\title{
Investigations of ice and emitter properties from radio signals recorded with ARIANNA
}

\author{
Robert Lahmann* for the ARIANNA Collaboration ${ }^{\dagger}$ \\ Friedrich-Alexander Universität Erlangen-Nuremberg ${ }^{\ddagger}$ \\ E-mail: robert. Iahmannefau.de
}

\begin{abstract}
ARIANNA is an in-ice radio detector for neutrinos in the energy range from $\sim 10^{16} \mathrm{eV}$ to $\sim 10^{20} \mathrm{eV}$ which is currently in its pilot-phase in Antarctica. It exploits the so-called Askaryan effect by which radio signals are emitted from neutrino-induced particle showers in ice. Geometric optics is an efficient tool to study the propagation of electromagnetic signals through a medium such as ice, using ray tracing to predict the response induced in an antenna for a given arrangement of emitter and receiver within the medium. Analyzing and understanding the antenna response to calibration signals under well-defined conditions is important to develop reconstruction algorithms of neutrino properties, most notably the interaction vertex and energy, and to understand and characterize the properties of the ice. Recent results obtained with the data collected during the 2018-2019 Antarctic season will be presented.
\end{abstract}

36th International Cosmic Ray Conference -ICRC2019-

July 24th - August 1st, 2019

Madison, WI, U.S.A.

\footnotetext{
* Speaker.

${ }^{\dagger}$ for collaboration list see PoS(ICRC2019)1177

$\ddagger$ currently at the University of California in Irvine
} 


\section{Introduction}

Given the strong reduction of the cosmic neutrino flux with increasing energy, radio detection in ice using the Askaryan effect [1] allows for a sparse instrumentation density, making it possible to survey a large volume for neutrino interactions in a very cost-efficient manner. The ARIANNA concept employs autonomous detector stations - each one comprising four or eight antennas located just slightly below the ice surface [2]. The concept of stations, referring to local clusters of antennas, is commonly used in designs of in-ice radio neutrino experiments. Other designs foresee antennas deployed at greater depths, but in general these depths are small compared to the thickness of the ice layer. Hence the effective volume must greatly exceed the instrumented volume in order to reach a significant detection rate for neutrinos. However, the vertical profile of the index of refraction in the medium leads to a downwards bending, i.e. away from the receivers at the surface, of the radio signals. This leads to the formation of a 'shadow zone' for an emitter at a given position in which receivers cannot be reached by radio signals. Nonetheless, signals have been observed in this shadow zone [3], which will be the main focus of this article.

The notion of a shadow zone results from the concept of geometric optics with the assumption of an index of refraction whose variation with depth can be described by a smooth analytical function. In practice, measurements for the index of refraction vs. depth $(z \leq 0)$ are fitted to the function

$$
n(z)=n_{\text {ice }}-\Delta n \exp \left(z / z_{0}\right)
$$

with the fit parameters $\Delta n$ and $z_{0}>0$, the scaling parameter. The asymptotic index of refraction of ice at large depth is $n_{\text {ice }}$ and $\Delta n=n_{\text {ice }}-n_{\mathrm{s}}$ with $n_{\mathrm{s}}$ being the index of refraction at the surface $(z=0)$. While the corresponding functions $n(z)$ derived for specific locations in Antarctica yield excellent predictions for the times of arrival (TOAs) in the 'refraction zone', i.e. the volume mutually exclusive with the 'shadow zone', it cannot explain the observations in the shadow zone. However, as the accumulation of snow that eventually solidifies into the Antarctic firn and ice is subject to seasonal environmental and climatic variations, a completely smooth variation of the index of refraction with depth would not be expected. While it is plausible that deviations of the vertical profile of the index of refraction from the smooth dependency described by Eq. (1.1) result in signals in the shadow zone, additional effects have been observed. These additional contributions to 'horizontal propagation' ${ }^{1}$ are most likely due to electric fields being formed at the boundary between firn and air which are not accounted for in geometric optics and need a full treatment using Maxwell's Equations. See [4] and references therein for a discussion.

Experimentally, horizontal propagation is of interest as it has the potential to increase the effective volume of a given setup of radio detectors. Furthermore, horizontal propagation may provide additional information about neutrino interactions: The emission pattern of the radio signal combined with the receiver topology leads to the experimental challenge of having to reconstruct the energy and interaction vertex of the neutrino interaction from the signals received by an individual station in the refraction zone. Additional signals from horizontal propagation - possibly in a different station - might help to better determine these parameters. Once the effects are understood

\footnotetext{
${ }^{1}$ In this article, for historical reasons all transmission modes by which signals reach a receiver in the shadow zone will be denoted 'horizontal propagation'.
} 
and can be parametrized, they can be implemented into Monte Carlo simulations without having to find computationally expensive numeric solutions to Maxwell's Equations for each simulation.

\section{Measurements}

The measurements described in this article were performed at the ARIANNA test site on the Ross Ice Shelf at a geographic location of about $79^{\circ} \mathrm{S}$ and $165^{\circ} \mathrm{E}$ during the 2018/19 Antarctic season. For data recording, an oscilloscope with sampling rate of $4 \mathrm{GSps}$ was used. An emitter was placed in a hole at $37.5 \mathrm{~m}$ (Vpol orientation) or $\sim 1 \mathrm{~m}$ (Hpol orientation) depth and a receiver was placed at distances of $25 \mathrm{~m}, 50 \mathrm{~m}, 75 \mathrm{~m}, 100 \mathrm{~m}, 300 \mathrm{~m}, 400 \mathrm{~m}, 500 \mathrm{~m}, 600 \mathrm{~m}$ and $935 \mathrm{~m}$. The depth of the receiver varied between about $1 \mathrm{~m}$ and $4 \mathrm{~m}$. For the measurements described in this article, for both emitter and receiver, dipole antennas were used. For the Vpol orientation, the dipole was oriented vertically, for the Hpol orientation, it was placed in the horizontal plane, perpendicular to the line of sight between emitter and receiver. Most measurements were done in the 'Vpol Vpol configuration', i.e. both emitter and receiver having Vpol orientation. For distances of $75 \mathrm{~m}$ and $300 \mathrm{~m}$, dedicated polarisation studies were performed with all four combinations of Vpol and Hpol orientation for emitter and receiver. In the shadow zone, $40 \sim 90$ signals were averaged for the analysis. Signal amplitudes are reported as 'effective voltage' $V_{\text {eff }}$, which is the oscilloscope voltage adjusted for the gain of amplifiers used in the shadow zone.

\subsection{Vpol - Vpol configuration}

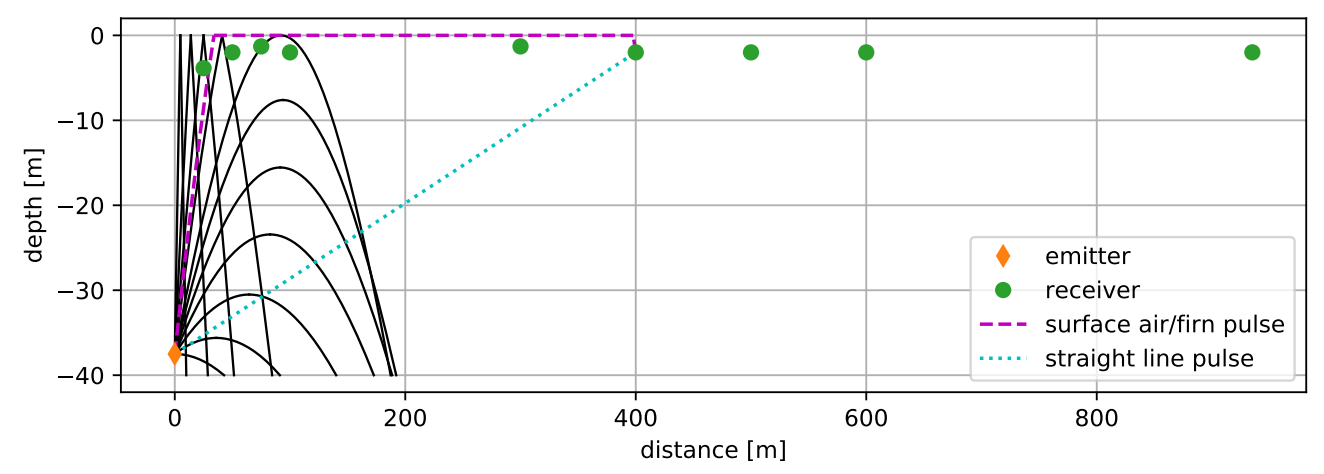

Figure 1: Depth vs. distance arrangement of emitter and receivers for measurements performed at the Ross Ice Shelf. The emitter is shown at the depth of the Vpol-Vpol configuration. The black lines indicate ray traces that result from the vertical profile of the index of refraction of the form given in Eq. (1.1). The receivers at distances of $300 \mathrm{~m}$ or further are in the shadow zone of the emitter. For the distance of $400 \mathrm{~m}$, the paths used to calculate the TOA of the surface air pulse, surface firn pulse and straight line pulse as described in Sec. 2.1 are shown.

Fig. 1 shows the experimental setup. Emphasis will be put on the shadow zone at distances of $300 \mathrm{~m}$ and more, where three distinct signal types are observed:

- 'Surface air pulse': The TOA is consistent with a ray travelling to the surface and reaching it at the critical angle, travelling in a straight line through the air along the surface and entering the firn at the critical angle such that it reaches the position of the receiver. 


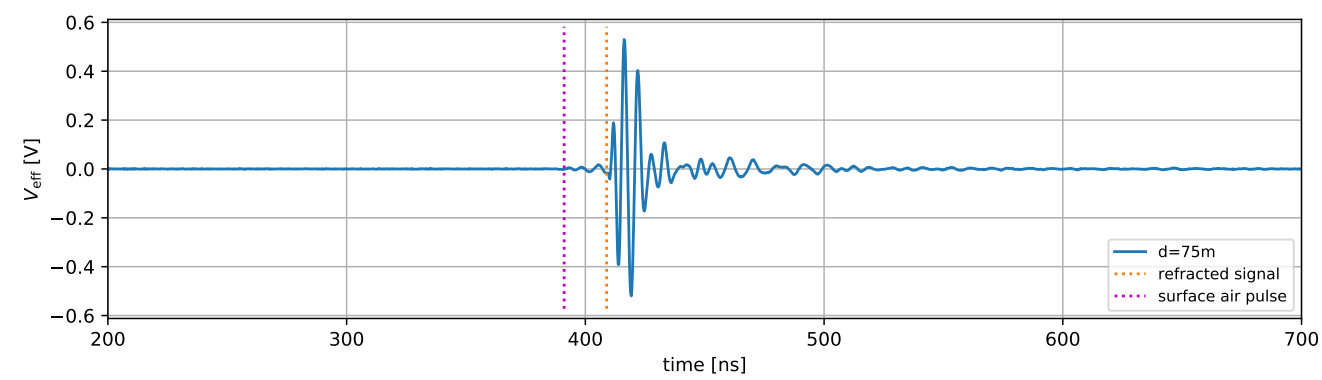

Figure 2: Time trace for signal in the refraction zone recorded at a horizontal distances of $75 \mathrm{~m}$ between emitter and receiver. The vertical line labelled 'refracted signal' indicates the expected TOA of the ray connecting emitter an receiver for a vertical profile of the index of refraction as given by Eq. (1.1). See text for explanation of the 'surface air pulse'.

- 'Surface firn pulse': As above, but the ray travelling slower in the firn along the surface.

- 'Straight line pulse': The TOA is consistent with the integral $c_{0}^{-1} \int n(z) d s$ with the speed of light in vacuum $c_{0}$ along the straight line path $s$ between emitter and receiver.

The corresponding paths are demonstrated for the distance of $400 \mathrm{~m}$ in Fig. 1. Note that these ray paths are not predicted by geometric optics, they rather are phenomenological description of the observation. The TOAs calculated in this fashion are overlaid with the time traces showing the respective types of pulse for a distance of $75 \mathrm{~m}$ in Fig. 2 and for the shadow zone in Fig. 3. In Fig. 2, only the TOA of the surface air pulse is given, as the surface firn pulse and straight line pulse superimpose with the much larger refracted signal. For a given distance, the error on the TOA is $\mathscr{O}(10 \mathrm{~ns})$ from the knowledge of the exact distance between emitter and receiver and the emission time of the pulse, affecting all calculated times at that distance in the same fashion.

The signals in the shadow zone (Fig. 3) show a rich structure. At $300 \mathrm{~m}$, all three pulses described above can be clearly separated and are in excellent agreement with the respective TOAs. Beyond that distance, the surface firn pulse and straight line pulse are spread out and merge into each other. In the following, the energy contents of the pulse types will be further investigated.

Fig. 4 shows the noise-subtracted energy spectral density (ESP) for the signals observed in the shadow zone within the time ranges of each type of signal shown in Fig. 3. The beginning of each time range was given by the respective TOA, the end was set by eye. Each of the three signal types shows a distinct frequency contents: The surface air pulse peaks around $100 \mathrm{MHz}$, the straight line pulse shows a broad frequency spectrum and the surface firn pulse peaks around $300 \mathrm{MHz}$. For the latter, in Fig. 3 the pulse cannot be distinguished by eye from the straight line pulse for the measurements beyond $300 \mathrm{~m}$, so the end point of the corresponding time interval was set to the calculated TOA of the straight line pulse, likely resulting in some overlap. In the logarithmic ESP-plot, however, for distances of $400 \mathrm{~m}, 500 \mathrm{~m}$, and $600 \mathrm{~m}$, a peak at $\sim 300 \mathrm{MHz}$ is visible.

Fig. 5 shows the measured energy in the time ranges for the respective signal types vs. distance. It can be seen that in addition to their frequency content, the signals also show differences in their attenuation with distance. The 'surface air pulse' has a slower attenuation with distance and starting at about $600 \mathrm{~m}$, it is the sole measurable component of the horizontal propagation signal. The exact mechanisms leading to the three different types of signals needs to be further investigated. 


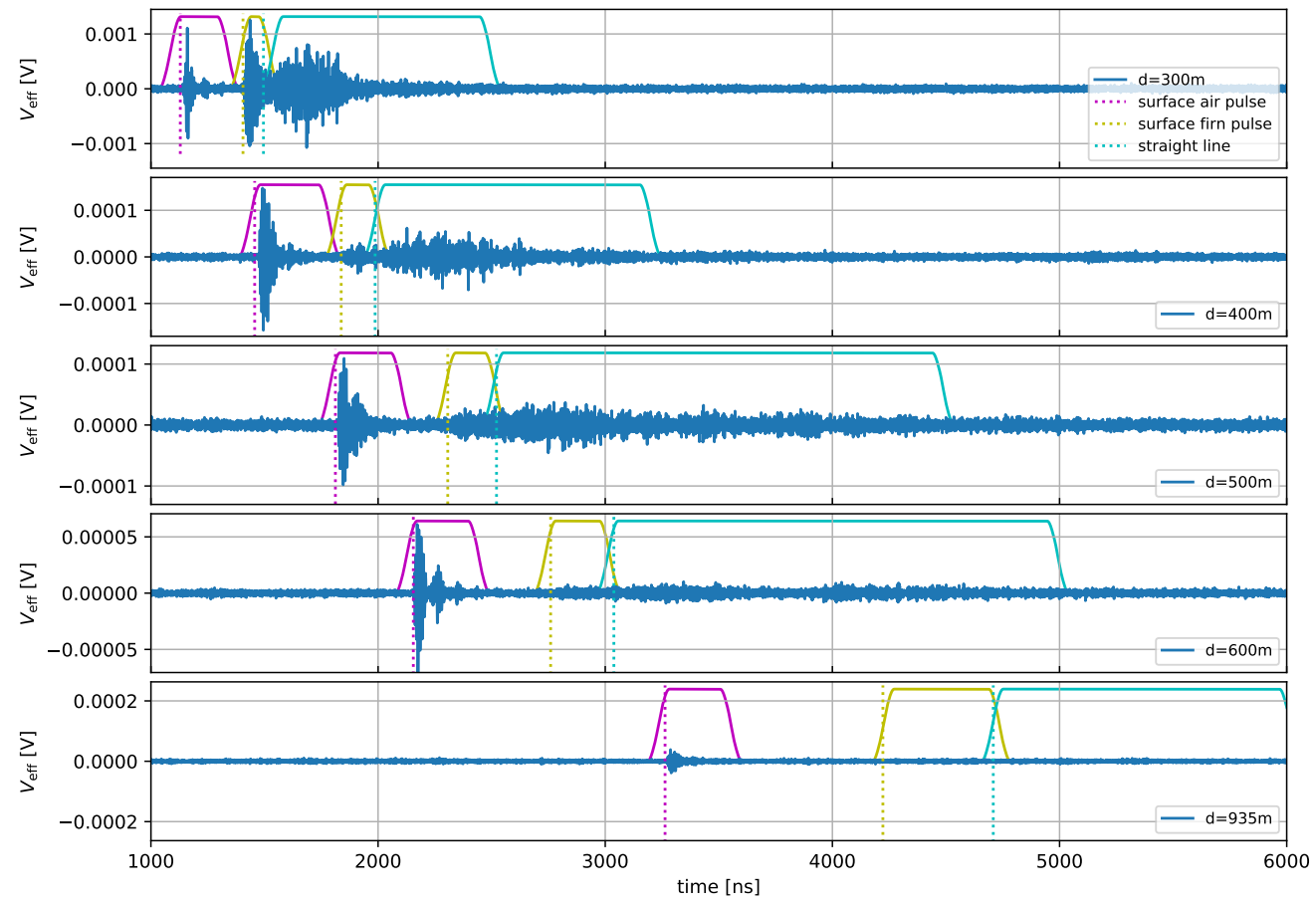

Figure 3: Time traces for signals in the shadow zone recorded at horizontal distances of $300 \mathrm{~m}, 400 \mathrm{~m}$, $500 \mathrm{~m}, 600 \mathrm{~m}$ and $935 \mathrm{~m}$ between emitter and receiver. Vertical lines indicate the expected TOAs of the respective signal types. The solid lines indicate the region spanned by $75 \%$-overlapping Hanning-windows, which were used to derive the energy and spectra associated with the respective pulse types. Windows were multiplied with some factor for visualisation in the plots to match the amplitude of the respective time traces.

As a means of assessing the absolute energy transmitted into the shadow zone, the energy of the pulse in the refraction zone at $d=100 \mathrm{~m}$ was scaled with $d^{-2}$ (assuming only geometric spreading of the energy) and with an additional absorption length $L_{E} \approx 220 \mathrm{~m}$ of the energy [5] $]^{2}$ for a frequency of $\sim 0.2 \mathrm{GHz}$. The energy of the pulses in the shadow zone can be seen to be about three to four orders of magnitude smaller than the reference energy including absorption. If a given signal were to originate from a neutrino, it would need an energy higher by a factor of $\sqrt{10^{3}} \sim \sqrt{10^{4}}=30 \sim 100$ in the shadow zone compared to the refraction zone in order to incite a radio signal with the same energy in an antenna at the same distance. Hence horizontal propagation has the potential to compensate the decreasing flux for higher neutrino energies by increasing the effective volume of a detector.

\subsection{Variation of dipole orientation and emitter depth}

Further measurements with the following configurations were performed:

- Horizontal distance of $75 \mathrm{~m}$ between emitter and receiver:

- Vpol emitter at $37.5 \mathrm{~m}$ depth ('deep emitter'), Vpol and Hpol receivers in refraction zone.

\footnotetext{
${ }^{2}$ Note that the absorption length given in that reference is for the amplitude of the electric field, which is twice as long as the absorption length for the energy of the signal. Effects stemming from the different density of ice and firn have been ignored for this qualitative study.
} 

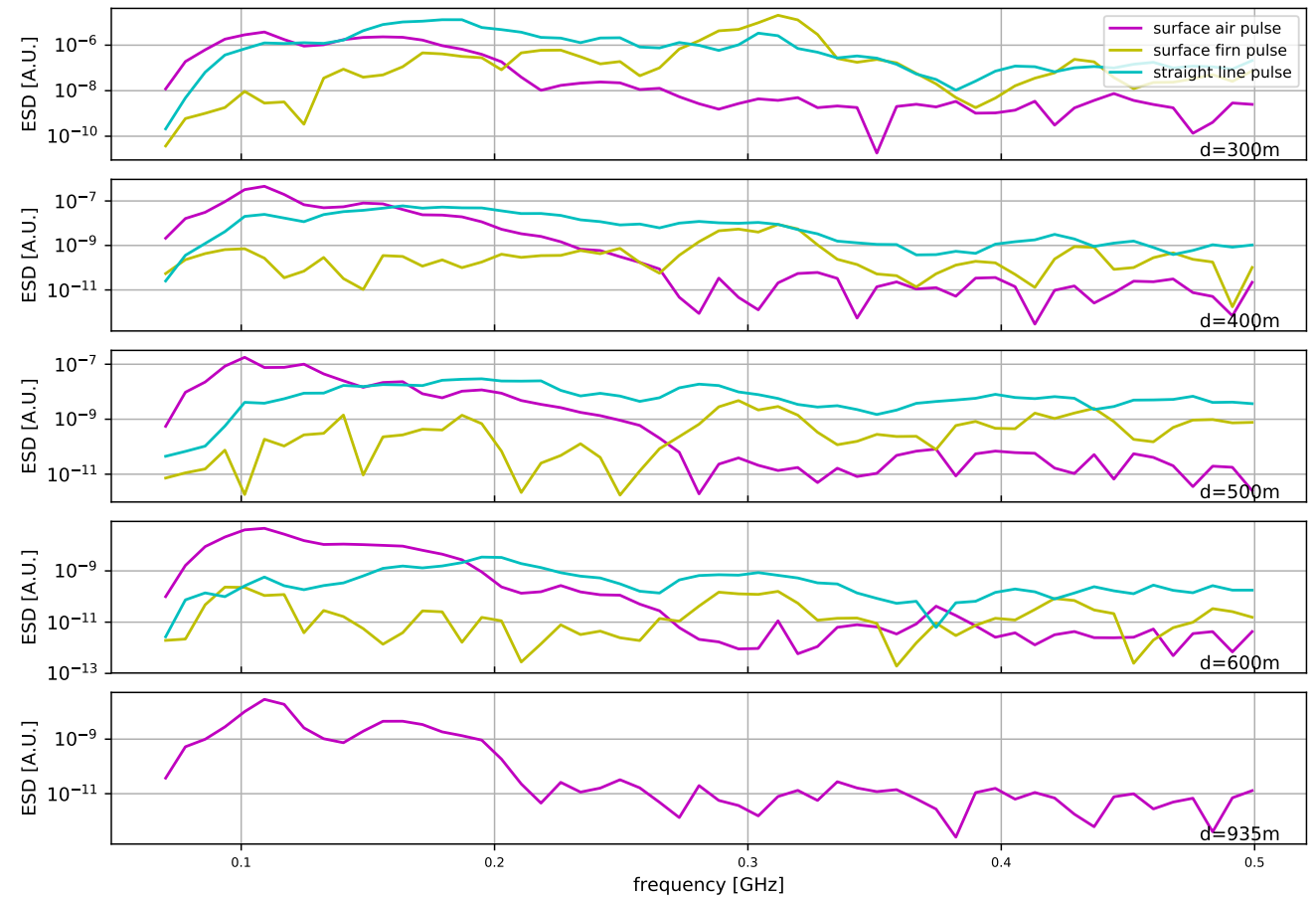

Figure 4: Energy spectral density (ESD) of signals in the time ranges depicted in Fig. 3 for the various distances between emitter and receiver in the shadow zone. The spectral noise distribution was estimated from the ESD of the time trace in a region without signal and subtracted from the ESD in the signal region. In the figure, the logarithm to the base 10 of the absolute value of this difference was plotted. As a negative value would be the result of statistical fluctuations, taking the absolute value results in positive values near zero that reflect the size of these fluctuations.

- Hpol emitter at $\sim 1 \mathrm{~m}$ depth ('shallow emitter'), Vpol and Hpol receivers in shadow zone ${ }^{3}$.

- Horizontal distance of $300 \mathrm{~m}$ between emitter and receiver:

- Vpol emitter at 37.5 m depth ('deep emitter'), Vpole and Hpol receivers in shadow zone.

- Hpol emitter at $\sim 1 \mathrm{~m}$ depth ('shallow emitter'), Vpol and Hpol receivers in shadow zone.

Of these combinations, the $75 \mathrm{~m}$ - 'deep emitter' configuration is the only one where the receiver is in the refraction zone. Here the signal energy for the cross-polarisation configuration is small, about $6 \%$ of the energy for the co-polarisation configuration, as would be expected.

The setups with shallow emitter and receivers in the shadow zone at $75 \mathrm{~m}$ and $300 \mathrm{~m}$, respectively, show a much brighter signal than expected, independent of the orientation of the receiver. The corresponding signals for $d=75 \mathrm{~m}$ are shown in Fig. 6. Despite of being in the shadow zone, the signal for the co-polar configuration (top plot in Fig. 6) carries about 50\% of the energy of the signal with the deep emitter and receiver in the refraction zone (cf. Fig. 2). The signal energy in the cross-polar configuration (bottom plot in Fig. 6) is about 4\% of that in the co-polar configuration. Note that the TOA of the surface air pulse seems to agree well with the data ${ }^{4}$, whereas the TOA of

\footnotetext{
${ }^{3}$ For technical reasons, the actual distance was $77 \mathrm{~m}$ for the Hpol-Hpol configuration.

${ }^{4}$ For the co-polar case (top plot in Fig. 6), the surface air pulse is hardly visible compared to the main pulse. In absolute terms, the surface air pulse for co- and cross-polarisation have roughly the same amplitude.
} 


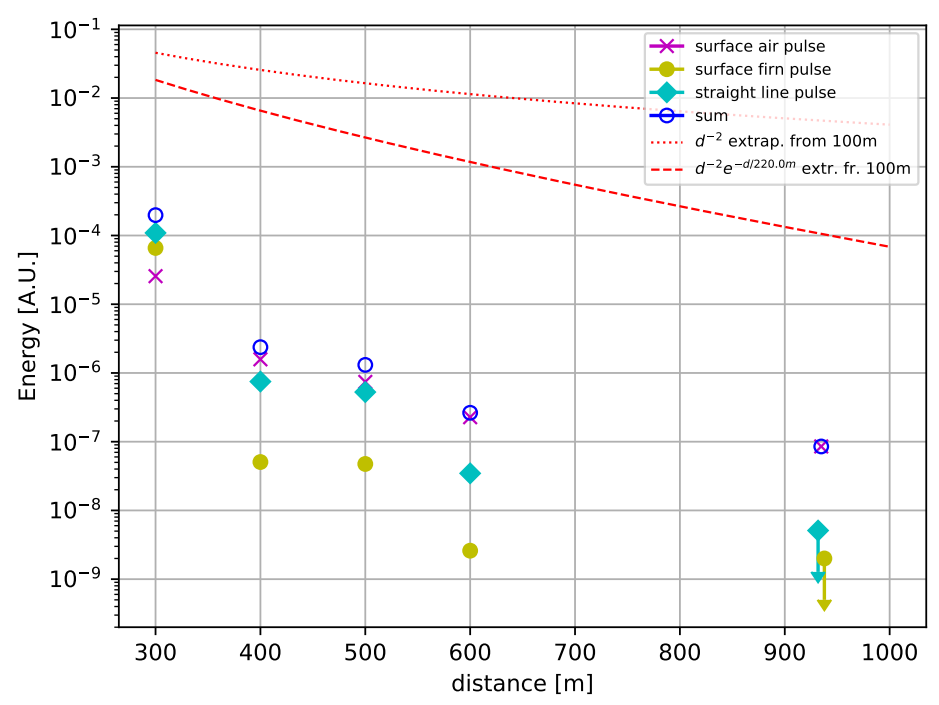

Figure 5: Energy vs. distance ( $=d$ in legend) for the energy in the different time ranges as obtained from the ESP in Fig. 4 for the Vpol-Vpol configuration. In addition, the sum of the energy in all time ranges is shown, corresponding to the total energy transmitted by horizontal propagation. For $d=935 \mathrm{~m}$, the energy for the surface firn pulse and straight line pulse is consistent with zero. As upper limits, $1 \sigma$ of the variation of the noise energy, when using different time windows for the calculation, was given. The extrapolations of the energy measured for the pulse in the refraction zone at $d=100 \mathrm{~m}$ allow for an assessment of the energy carried by the pulses in the shadow zone. This is discussed in more detail in the text.

the straight line pulse does not coincide with the onset of the main signal. This seems to indicate that channelling effects are at play: As the emitter and receivers are roughly at the same depth, it is conceivable that rays get trapped efficiently between the surface and some layer with a different (smaller) index of refraction below emitter and receiver.

For the straight line signal of the deep emitter at $300 \mathrm{~m}$ distance, the cross-polarization and copolarization signals have roughly the same energy. Possibly the origin of the 'straight line signal' is multiple 'diffuse refraction' and 'diffuse reflection', i.e. a spread of signal power around the angles predicted by Snell's law, at layers with discrete steps in the index of refraction. This process could lead to an eventual 'randomization' of the polarisation at the location of the receiver. The 'true' path in the picture of geometric optics would then be a succession of curved paths for which in total the travel time is roughly similar to the hypothetical travel time for a straight line signal.

\section{Summary and Conclusions}

Data recorded for various configurations of dipole radio emitters and receivers during the 2018/19 Antarctic season at the site of the ARIANNA detector on the Ross Ice Shelf were presented. Three types of pulses could be discerned for the Vpol-Vpol configuration of emitter and receiver in the 'shadow zone' that differ in their travel time, frequency contents and attenuation with distance. The measured TOAs of the respective pulses could be well reproduced with a phenomenological description for specific ray paths between emitter and receiver. For neutrino 


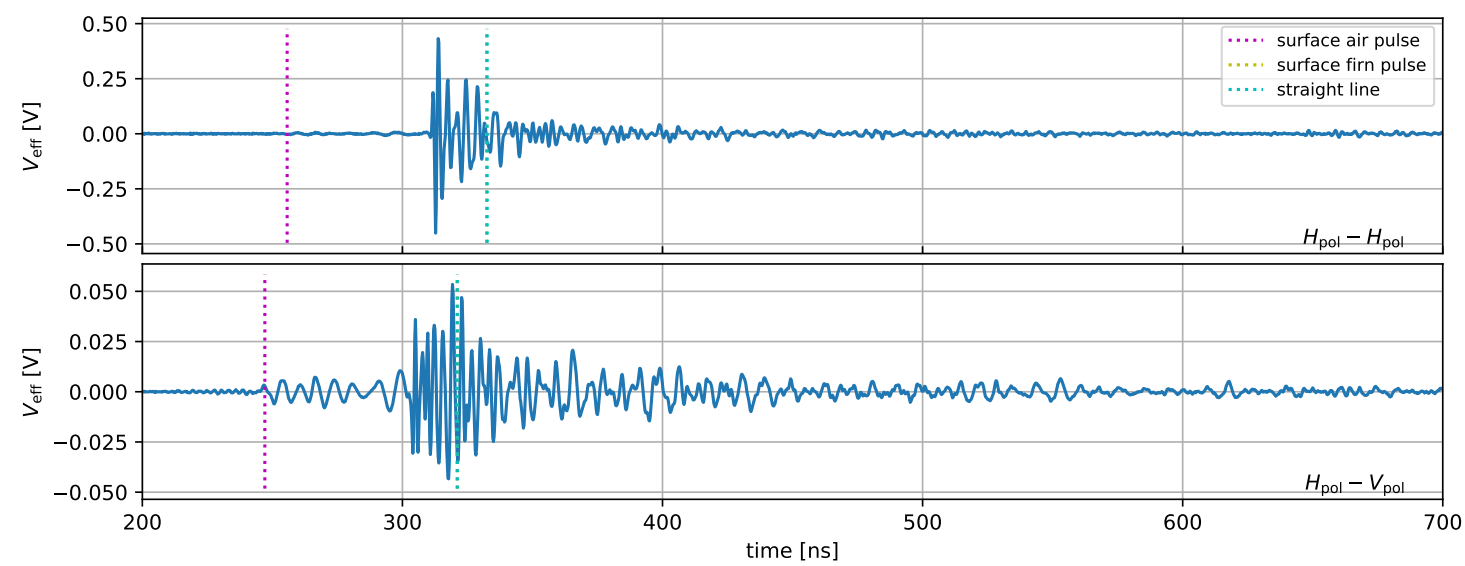

Figure 6: Time traces for signals in the shadow zone recorded at horizontal distances of $77 \mathrm{~m}$ (top) and $75 \mathrm{~m}$ (bottom) between emitter and receiver for an Hpol emitter at $2 \mathrm{~m}$ depth for co-polarized (top) and cross-polarised (bottom) receiver. Vertical lines indicate the expected TOAs of the respective signal types.

detection, the observed effects could increase the effective volume significantly for neutrinos with energies exceeding the trigger threshold for signals in the refraction zone by a factor of $30 \sim 100$.

Further effects with much larger amounts of energy transmitted into the shadow zone were observed which seem to be specific to particular configurations of emitter and receiver in a given volume of ice and firn. One likely effect is 'channelling', i.e. the firn acting as a (leaky) wave guide when both emitter and receiver are located in a layer of firn between the surface and another layer of firn with a discrete change of the index of refraction. Further studies are necessary to derive a genuine model of 'horizontal propagation' that can be implemented into Monte Carlo simulations.

\section{Acknowledgements}

We are grateful to the U.S. National Science Foundation-Office of Polar Programs, the U.S. National Science Foundation-Physics Division (grant NSF-1607719), the U.S. Dept. of Energy, and the Taiwan Ministry of Science and Technology. R.L. thanks UC Irvine and the FAU ErlangenNürnberg for supporting his research stay at UCI.

\section{References}

[1] G.A. Askariyan, Excess Negative Charge of an Electron-Photon Shower and its Coherent Radio Emission, Sov. Phys. JETP 14 (1962) 441.

[2] A. Anker et al., Targeting ultra-high energy neutrinos with the ARIANNA experiment, Advances in Space Research (2019) .

[3] S.W. Barwick et al., Observation of classically 'forbidden' electromagnetic wave propagation and implications for neutrino detection, JCAP 07 (2018) 055.

[4] C. Deaconu et al., Measurements and Modeling of Near-Surface Radio Propagation in Glacial Ice and Implications for Neutrino Experiments, Phys. Rev. D 98 (2018) 043010.

[5] J.C. Hanson et al., Radar absorption, basal reflection, thickness and polarization measurements from the Ross Ice Shelf, Antarctica, J. Glaciol. 61 (2015) 438. 